\title{
Double siRNA-targeting of CIAP2 and LIVIN results in synergetic sensitization of HCT-I I 6 cells to oxaliplatin treatment
}

This article was published in the following Dove Press journal:

OncoTargets and Therapy

20 September 2013

Number of times this article has been viewed

\author{
Andrey S Bavykin' \\ Alexandra A Korotaeva' \\ Stanislav V Poyarkov ${ }^{2}$ \\ Alexandr V Syrtsev ${ }^{1,3}$ \\ Sergei A Tjulandin ${ }^{3}$ \\ Alexandr V Karpukhin' \\ 'Russian Academy of Medical Science \\ Research Centre for Medical \\ Genetics, Moscow, Russian Federation; \\ ${ }^{2}$ D.I. Ivanovsky Institute of Virology \\ Russian Academy of Medical Sciences, \\ Moscow, Russian Federation; ${ }^{3}$ N.N. \\ Blokhin Russian Cancer Research \\ Center under the Russian Academy \\ of Medical Sciences; Moscow, Russian \\ Federation
}

Purpose: Most colon cancers show low sensitivity to treatment with oxaliplatin and a specific strategy is needed to overcome this problem. Our approach uses RNA interference to silence the expression of target genes responsible for the development of oxaliplatin resistance. Profile analysis of genes related to the regulation of apoptosis allowed identification of target genes showing the greatest degree of upregulation in response to oxaliplatin exposure.

Methods: We designed a panel of genes with functions closely related to inactivation of the caspase cascade, endoplasmic reticulum stress reduction, and drug metabolism. The candidate genes were silenced by means of specific small interfering RNA (siRNA) oligonucleotides.

Results: The caspase 3 and 9 inhibitors of apoptosis 2 (cIAP2) and LIVIN were found to be the most dose-responsive genes during the period of oxaliplatin treatment. Two-fold sensitization of cells to oxaliplatin was observed with independent knockdown of either cIAP2 or LIVIN expression. siRNA-silencing of both targets produced a five-fold increase in oxaliplatin sensitivity of HCT-116 cells.

Conclusion: A dose-dependent approach revealed reliable targets for siRNA-silencing under low doses of oxaliplatin. Targeting the key proapoptotic chain with several specific siRNAs resulted in synergetic sensitization of HCT-116 cells to oxaliplatin treatment.

Keywords: drug resistance, inhibition of apoptosis, RNA interference

\section{Introduction}

Following the introduction of the drug oxaliplatin in colorectal cancer therapy, $40 \%-50 \%$ of patients have shown improved response rates and prolonged survival. However, more than half of patients experience side effects and drug resistance and new therapeutic approaches are needed.

Many studies ${ }^{1-4}$ have searched for candidate target genes that express response to oxaliplatin treatment. Recent investigations using array technology have revealed a number of candidate gene clusters involved in the cell cycle, DNA replication, DNA transcription, and apoptosis. ${ }^{5,6} \mathrm{We}$ focused on the caspase pathways in order to identify the most responsive markers related to oxaliplatin treatment. The activity of caspases is regulated through a variety of apoptotic inhibitors, including such protein families as baculoviral IAP repeat-containing (BIRC) proteins, Bcl-2, heat-shock proteins (HSPs), glucose-regulated proteins (GRPs), tumor necrosis factor receptor (TNFR)related proteins (such as TRAP1), etc. The expression panel used in our investigation contained direct inhibitors of caspases (cIAP1/2, XIAP, Survivin, and LIVIN), CFLAR inhibitors of reactive oxygen species (ROS)-induced apoptosis (TRAP1, Bcl-xl, $B c l 2$, and Grp78), and several genes involved in drug metabolism (Gstp1, Gstm2,
Correspondence: Andrey Bavykin Russian Academy of Medical Science Research Centre for Medical Genetics, Moskvorechie Street I, Moscow, Russian Federation, 115479

Tel +74993241239

Fax +7 4993240702

Email bavykinas@mail.ru 
and endonuclease excision repair cross-complementing 1 [ERCC1]) as representative of the DNA reparation system. Together, these genes are involved in the mechanism that prevents apoptosis of cancer cells caused by either drug treatment or other extrinsic stress factors. Although several of these proteins have been previously used as the targets of RNA interference (i) knockdown in different cancer cell types, ${ }^{2,3,7,8}$ their role in chemotherapy resistance is still not clearly understood. Our approach was to study time- and dose-dependent responses of the HCT-116 cell line to oxaliplatin exposure by means of an expression panel of apoptotic inhibitors consisting of different protein families. Our study has revealed at least four genes that are upregulated by oxaliplatin doses of 5 and $10 \mu \mathrm{M}$, of which CIAP2 and LIVIN appeared to be the most dose-responsive during incubation with oxaliplatin. The caspase 3 and 9 inhibitor $c I A P 2$ is a well-known $c-M y c$ target and is frequently elevated in lung, colon, and pancreatic cancers, while there is relatively no information about Birc7/LIVIN. Knockdown of both LIVIN and $C I A P 2$ resulted in dramatic levels of apoptosis at $10 \mu \mathrm{M}$ of oxaliplatin. This observation suggests a novel synergistic role of these genes in the regulation of apoptosis and the development of oxaliplatin resistance.

\section{Materials and methods Cells}

Colon cancer HCT-116 p53 $3^{-/}$cells were a gift from Professor AA Shtill, N.N. Blokhin Cancer Research Center, Moscow, Russian Federation and were maintained in Dulbecco's Modified Eagle's Medium ([DMEM] Life Technologies, Carlsbad, CA, USA) supplemented with $10 \%$ fetal bovine serum ([FBS] Life Technologies) and $50 \mu \mathrm{g}$ of penicillin-streptomycin at $37^{\circ} \mathrm{C}$ in $5 \% \mathrm{CO}_{2}$.

\section{Oxaliplatin treatment and real time-PCR}

The cells were seeded in 6-well plates in DMEM and 5\% FBS without antibiotics, so that they will give 50\% confluence on the next day. Cells were exposed to 5 and $10 \mu \mathrm{M}$ oxaliplatin (Pharmachemie BV, Haarlem, the Netherlands) for 24 and 48 hours and real time- PCR was performed to analyze gene expression. Untreated cells served as a negative control.

\section{Cell viability and apoptosis}

Cell viability was determined by Trypan blue and apoptosis assay staining. Staining for the apoptosis analysis was performed using a Vybrant Apoptosis Assay Kit \#5 (Life Technologies) with Hoechst 33342/propidium iodide, according to the manufacturer's instructions. Cells were viewed and counted using an Axio Observer D1 microscope (Carl Zeiss Meditec AG, Jena, Germany) with $10 \times / 20 \times$ objective lenses. Images were captured using a Carl Zeiss AxioCam MRc camera. Tests were performed in triplicate, counting a minimum of 600 cells total in each.

\section{RNA isolation, reverse transcription}

In order to perform reverse-transcription-PCR for all samples in equivalent conditions, RNA was isolated from each experimental well using an RNeasy Mini Kit (Qiagen, Hilden, Germany) and cells from triplicate wells were combined. Lysis buffer was added to the wells with the cells and incubated for 15-20 minutes until all cells were lysed. Isolation was performed according to the manufacturer's instructions and the RNA concentration was measured on a NanoDrop 1000 (Thermo Fisher Scientific, Waltham, MA, USA). One hundred to five hundred nanograms of total RNA was applied for RT in a total volume of $20 \mu \mathrm{L}$ using $1-5 \mathrm{U}$ of Promt II reverse transcriptase (Promega Corporation, Fitchburg, WI, USA) at $42^{\circ} \mathrm{C}$ for 1 hour. The reaction was stopped by heating at $70^{\circ} \mathrm{C}$ for 10 minutes.

\section{Real-time PCR}

Expression profiles of the genes c-IAP1, cIAP2, XIAP, Survivin, LIVIN, FLIP (L-long chain splice variant), $c-M y c$, Grp78, 1-Bcl-2, Bcl-xl, Gstp1, Gstm2, Gstm4, ERCC1, and TRAP1 were determined real time-PCR using a StepOne Real-Time PCR System (Life Technologies) with EveGreen (Biotium, Hayward, CA, USA). Primers were designed according to standard rules in order to amplify 200-220 basepair fragments of all analyzed genes. The primer sequences used for amplification were as follows:

- IAP-1F ( $c I A P 1$ forward): AGGTGTGAGTTCTTGATACGAA

- IAP-1R(cIAP1 reverse) : TTGTTTCACCAGGTCTCTATTA

- Bir3-F (cIAP2 forward): AGGTGTTGGGAATCTGGAGAT

- Bir3-R ( cIAP2 reverse): GCAGCATTAATCACAGGAGTA

- XF (XIAP forward): TAGGTGAAGgTGATAAAGTAA

- XR (XIAP reverse): TTCTAGTTAGTGATGGTGTT

- Bir5F (Birc 5 forward): CCCAGTGTTTCTTCTGCTT

- Bir5R (Birc 5 reverse): GGCTCTTTCTCTGTCCAGTT

- Liv-F(LIVIN forward): TGCCTGTTCTGGACTGTGT

- Liv-R (LIVIN reverse): GCATCCAACAGGTACAGTT

- LF1 (Long FLIP forward): TAATGGGAGAAGTAAAGAACAA

- LF2: (Long FLIP reverse) AGGGAAGTGAAGGTGTCTCGAA

- $\mathrm{CMF}$ ( $c-M y c$ forward): GAGGCTATTCTGCCCATTTG

- CMR ( $c-M y c$ reverse): TCCTCGTCGCAGTAGAAATAC

- Grp-F (Grp78 forward): AGGTGGGCAAACAAAGACAT 
- Grp-R (Grp78 reverse): CCGTAGGCTCGTTGATGAT

- Bcl-2F (Bcl-2 forward): GGATTGTGGCCTTCTTTGAGT

- Bcl-2R ( $B c l-2$ reverse): TCAGAGACAGCCAGGAGAAA

- BL-F ( $B c l-x L$ forward): GAGGCAGGCGACGAGTTTGA

- BL-R ( $B c l-x l$ reverse): ACAGTCATG CCCGTCAGGA

- Gstp-F (Gstpl forward): CATCTCCCTCATCTACACCA

- Gstp-R (Gstp1 reverse): TCACTGTTTCCCGTTGCCA

- Trp-F (Trap1 forward): GATGGTTCT GGAGTGTTTGA

- Trp-R (Trap1 reverse): GTG TTCATCCGCCTTCCATTC

- GSTM2-F: AGGAGCAGATTCGCGAAGACAT

- GSTM2-R: TGTGAGTAGAGCTTCAGCATT

- GSTM4-F: CAACGCCATCCTGTGCTACAT

- GSTM4-R: GCTGCATC atTGtagGaAgtT

- ERC-F1(ERCC1 forward): CACAACCTGCACCCAGACTA

- ERC-R1(ERCCl reverse) : TCCGCTGGTTTCTGCTCATA

- GH-F (GAPDH forward): GAACCATGAGAAGTATGACAA

- GH-R (GAPDH reverse): TGAGTCCTTCCACGATACCAA

The real time-PCR conditions were as follows: one cycle of initial denaturation at $95^{\circ} \mathrm{C}$ for 15 minutes, 42 cycles of amplification at $95^{\circ} \mathrm{C}(25$ seconds $), 60^{\circ} \mathrm{C}$ ( 25 seconds $)$, and $72^{\circ} \mathrm{C}$ ( 5 seconds); and a melting step of $95^{\circ} \mathrm{C}$ for 1 minute, and an increase from $60^{\circ} \mathrm{C}$ to $95^{\circ} \mathrm{C}$, with 1 minute for each degree. The GAPDH gene was amplified as a control with each group of genes at the corresponding annealing temperature. All experiments were repeated at least three times.

\section{Small interfering RNA (siRNA) design}

siRNA oligonucleotides that knock down messenger (m) RNAs of the genes $C I A P 2$ and LIVIN and non-targeting (scrambled) siRNAs were designed using the program BLOCK-iT'TM RNAi Designer (Life Technologies). Tuschl's motif was used as the criterion for the target sequence search. We used an "AA(N19)TT" pattern, so that the tool searched for an area of the sequence that begins with "AA" and ends with "TT," with 19 bases in-between. Several variants of each of the 21-22 bp-long siRNAs were designed for each target, and the BLOCK-iT program was used to identify the most sensitive. siRNA oligonucleotides were synthesized in sense and antisense strands in order to produce duplexes resistant to intracellular nucleases. The RNA oligonucleotides were synthesized with two flanking deoxyribonucleotides (dTdT) at the $3^{\prime}$ end. The sequences of the siRNA oligonucleotides were as follows:

- $\quad$ cIAP2: sense, UAAGGGAAGAGGAGAGAGAAdTdT; antisense, UUCUCUCUCCUCUUCCCUUAdTdT

- LIVIN: sense, CUGUACCUGUUUGGAUGCUdTdT; antisense, AGCAUCCAAACAGGUACAGdTdT
- Scrambled: sense, CCUGGUAGCAGCGAGUGAGdTdT; antisense, CUCACUCGCUGCUACCAGGdTdT.

\section{Statistical analysis}

Statistical analysis was carried out using an unpaired Student's $t$-test (GraphPad Software Inc, La Jolla, CA, USA). Results were considered statistically significant where $P<0.05$.

\section{Transfection, siRNA interference, and oxaliplatin treatment}

Cells were seeded for transfection in 48-well plates in DMEM without antibiotics 1 day before transfection so that they were $55 \%-60 \%$ confluent on the day of transfection. For transfection, the regular medium was replaced with low-serum medium with $1 \%$ FBS without antibiotics. The cells were transfected with siRNA in a forward manner using Lipofectamine RNAiMAX (Invitrogen; Life Technology). The final concentrations of the siRNA mixtures contained two equimolar siRNAs in concentrations up to $50 \mathrm{nM}$, as recommended in the RNAiMAX instruction protocol. Knockdown efficiency was examined using real time-PCR, performed as previously stated. Oxaliplatin $(10 \mu \mathrm{M})$ was added to each well containing the siRNA transfected cells. Untreated cells with no siRNA or oxaliplatin were used as negative controls; cells treated with oxaliplatin only were used as an siRNA negative control; and cells treated with scrambled siRNA were used as a scrambled control. The cells were incubated with the siRNA-Lipofectamine RNAiMAX complexes and oxaliplatin for 48 hours, and apoptosis was analyzed by fluorescent microscopic examination.

\section{Results \\ Gene expression in HCT-I 16 cells in response to dosage and timing of oxaliplatin treatment}

To determine the level of the expression response to different doses of oxaliplatin, HCT-116 cells were plated $\left(2 \times 10^{5}\right.$ per well on a 48-well plate) and grown with the addition of oxaliplatin. In most cases, the effect of siRNA inhibition appeared after 48-72 hours or more. However, some cell lines did not survive during the long period of treatment with oxaliplatin. We incubated the HCT-116 cells with oxaliplatin for $24,48,72$, and 96 hours (Figure 1). We observed that 48 hours of treatment with oxaliplatin is the optimal period for evaluating the apoptotic effect produced by RNA interference; a longer period results in more than $30 \%$ cell death, which is equivalent to the effect of a single siRNA. Furthermore, a strong apoptotic response makes it difficult to perform accurate measurements of mRNA expression. Oxaliplatin at $5 \mu \mathrm{M}$ and $10 \mu \mathrm{M}$ was added to each 


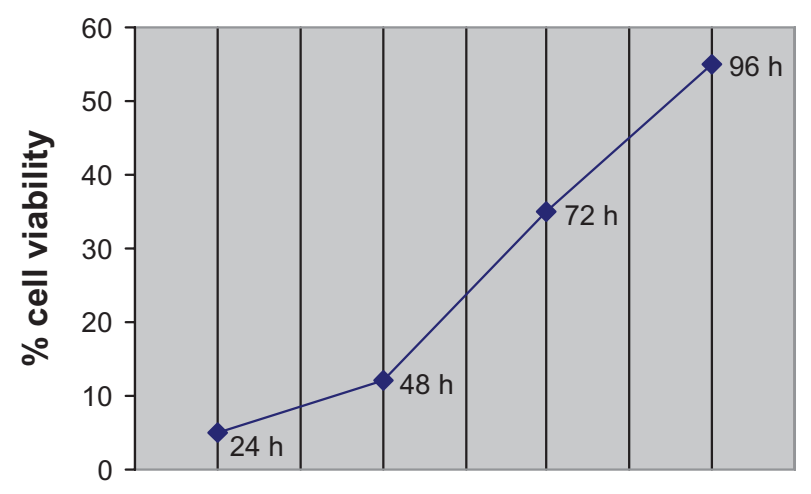

Figure I Viability of HCT-II 6 cells that were treated with $10 \mu \mathrm{M}$ of oxaliplatin. Notes: HCT-I 16 cells were treated with $10 \mu \mathrm{M}$ of oxaliplatin and incubated for 24-96 hours (h) in Dulbecco's Modified Eagle's Medium with I\% fetal bovine serum. Viability was identified by Trypan blue. well, with triplicate wells for each concentration. An incubation period of 48 hours is commonly believed to be sufficient, as by this point all plated cells will have passed through the division cycle, and most would be able to express early ( 24 hours of oxaliplatin treatment) and late (48 hours) genes in response to oxaliplatin treatment. After 24 and 48 hours of incubation, real time-PCR analysis was performed. The profile expression results are described in Figure 2 and correspond to the classic apoptosis network shown in Figure 3.

\section{Early genes}

TRAP1, XIAP, and ERCC1 showed the strongest early increase in expression (more than two-fold) at both concentrations of

A

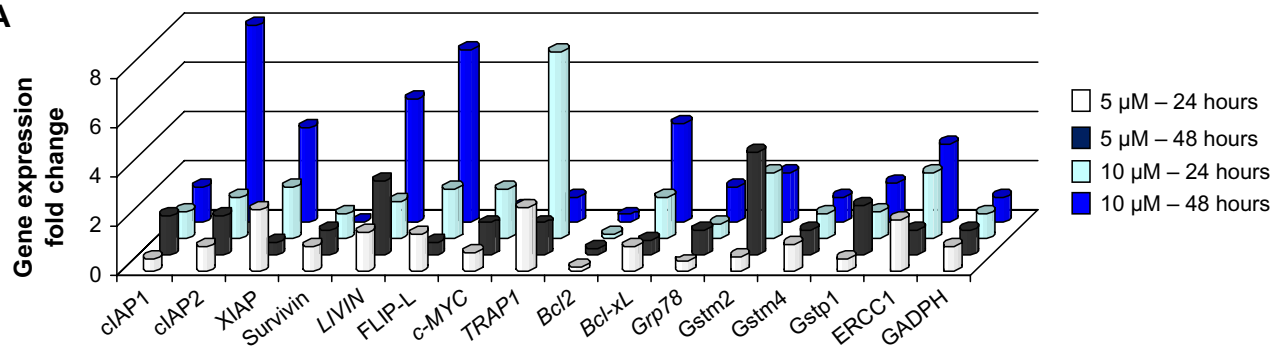

B

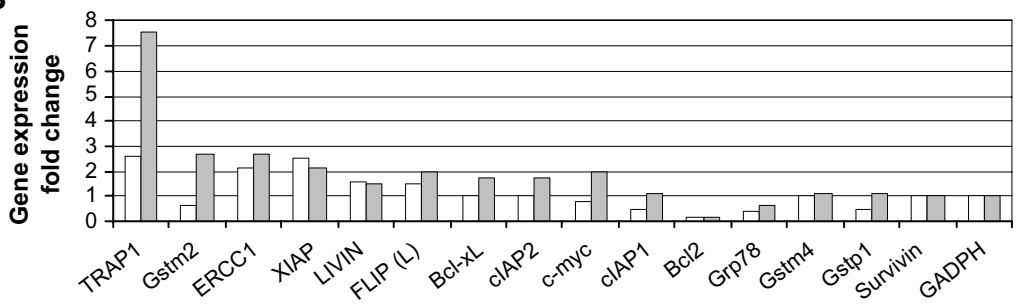

$\square 5 \mu \mathrm{M}-24$ hours

$\square 10 \mu \mathrm{M}-24$ hours

C

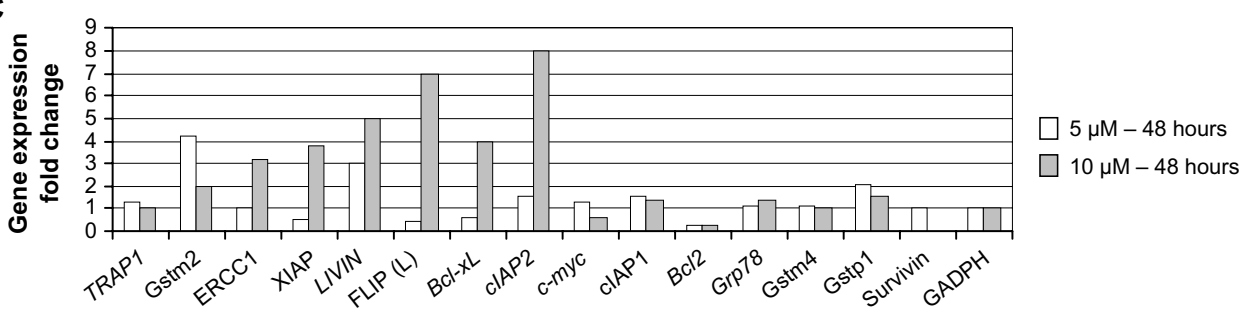

D

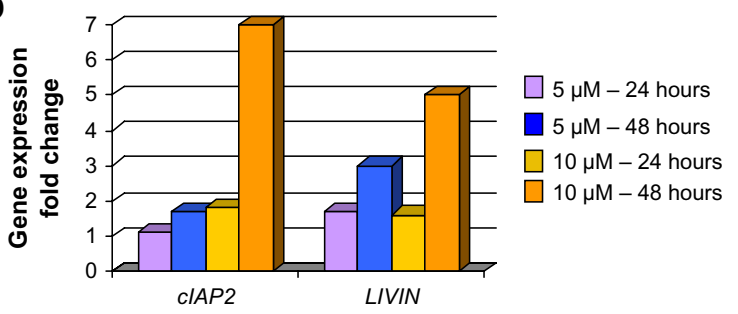

Figure 2 Dose- and time-dependent gene expression profiles.

Notes: HCT-116 cells were treated with 5 or $10 \mu \mathrm{M}$ of oxaliplatin. Fold changes were determined by $\Delta \Delta \mathrm{Ct}$ relative quantification method. Calculation of fold changes was performed using a formula $2^{-\Delta \Delta C t}$, where " 2 " is a fold change in amplicon between cycles and $\Delta \Delta \mathrm{Ct}$ the normalized cycle change between sample and reference. Samples are the genes from the cells that were treated with oxaliplatin, and reference are the genes from the untreated cells. Gene GAPDH (glyceraldehyde-3-phosphate dehydrogenase human) was used as the endogenous control. (A) Gene expression in dose-dependent analysis during the period of 48 hours of oxaliplatin treatment. (B) Dose-dependent profiles of early genes (24 hours). (C) Dose-dependent profiles of late genes (48 hours). (D) LIVIN and cIAP-2 were chosen as the candidate genes for small interfering RNA (siRNA) knockdown. 

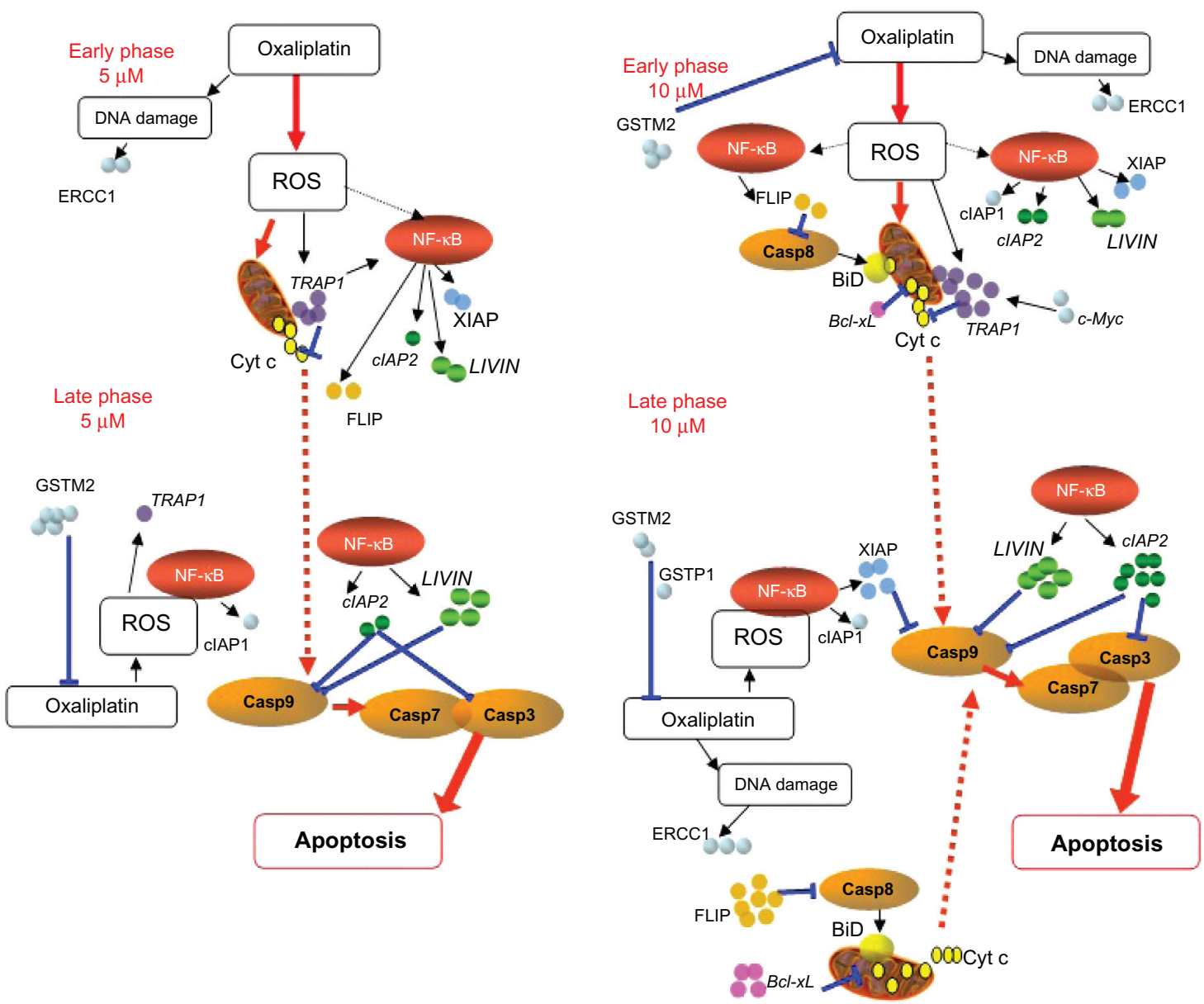

Figure 3 Early and late phases of gene expression under 5 and $10 \mu \mathrm{M}$ of oxaliplatin.

Notes: In small circles are the expressions of genes measured by means of real-time PCR (except cytochrome [cyt] c [yellow]). Quantity of circles at all stages correlates with the measured level of gene expression. Large ellipses depict NF-KB signaling and caspase (casp 8, 9, 7, 3) cascades, that were implicated in either regulation of gene expression or targeting. Black and red arrows indicate activation, while blue blocking arrows indicate inhibition processes.

Abbreviations: PCR, polymerase chain reaction; ROS, reactive oxygen species; NF- $\mathrm{KB}$, nuclear factor kappa-light-chain-enhancer of activated B cells.

oxaliplatin, and slight increases were observed for $c-M y c$ and Gstm 2 at $10 \mu \mathrm{M}$ of oxaliplatin (Figures 2B and 3). Early expression of LIVIN and CIAP2 genes was intermediate (1.2-1.7×). Upregulation of TRAP1,ERCC1, and Gstm2 was found to be strongest at $10 \mu \mathrm{M}$ of oxaliplatin; however, none of these genes were upregulated for the entire period of drug exposure. TRAP1 is believed to localize to the cytosolic and mitochondrial fractions. The functional role of TRAP1 in mitochondria has been shown by a number of studies, ${ }^{9,10}$ in which it has been described as a negative mediator of cytochrome $\mathrm{c}$ release (Figure 3 ) and downstream activation of caspase 9. Subsequent activation of the caspase 9 and 3 inhibitors LIVIN and CIAP2 seems to support the consistency of such events. Early activation of TRAP1 may occur as a response to drug metabolism that triggers activation of the nuclear factor kappa-light-chainenhancer of activated B cells (NF- $\mathrm{KB}$ ) signaling pathway ${ }^{11}$ and subsequent activation of XIAP, which stops apoptotic cell death by direct inhibition of caspases 3, 7, and 9 (Figure 3).

\section{Late genes}

Among the late genes, the targets of NF- $\mathrm{KB}$ signaling, the caspase inhibitors cIAP1, CIAP2, XIAP, and FLIP, ${ }^{12,13}$ appeared to be upregulated, mostly at $10 \mu \mathrm{M}$ of oxaliplatin (Figures $2 \mathrm{C}$ and 3). LIVIN and CIAP2 are believed to inhibit apoptosis downstream of the caspase cascade by direct inhibition of both caspases 3 and 9. FLIP was among the most highly upregulated late genes at $10 \mu \mathrm{M}$ of oxaliplatin. This event correlated with upregulation of another apoptotic inhibitor, $B c l-x l$, under the same time/ dose conditions, and was likely due to extension of the ROS influence and NF- $\kappa \mathrm{B}$ signaling because of increased concentrations of the drug.

\section{Oxaliplatin-dependent expression}

Within 48 hours at $5 \mu \mathrm{M}$ of oxaliplatin, elevated rates of expression were observed for LIVIN, Gstm2, and Gstp1. However, at $10 \mu \mathrm{M}$ of oxaliplatin, we observed a twofold 
reduction of Gstm2 and downregulation of Gstp1. Within 48 hours at $10 \mu \mathrm{M}$ of oxaliplatin, increased rates of expression were observed for $C I A P 2$ (four-fold increase), LIVIN (three-fold), and FLIP, Bcl-xl, and XIAP (two-fold). TRAP1 and Survivin were significantly downregulated during this period. Upregulation of FLIP and ERCC1 was found to be time-dependent at the higher dose of oxaliplatin, while at $5 \mu \mathrm{M}$, downregulation of these genes was observed in 48 hours.

\section{Time- and dose-dependent genes}

Birc 7/LIVIN was the only gene that appeared to exhibit stable elevated expression within 48 hours at the lower concentration and was dose-dependent at the later stage. The expression of $C I A P 2$ appeared to be both time- and dose-dependent (Figures 2D and 3). We chose to further investigate LIVIN and cIAP2 (Figure 2D), as two direct inhibitors of pro-caspase 3 and 9 , in order to determine whether double siRNA knockdown of two key apoptosis regulators may influence cell resistance to oxaliplatin.

\section{siRNA silencing of the candidate genes}

Knockdown of target genes was performed at the concentration of oxaliplatin $(10 \mu \mathrm{M})$ that caused the greatest elevation in their expression levels (Figure 2A). For $C I A P 2$, the maximum rate of silencing was obtained in 48 hours by means of $15 \mathrm{nM}$ of siRNA and was as much as $80 \%(P<0.024)$ without oxaliplatin and $65 \%$ when $10 \mu \mathrm{M}$ of oxaliplatin was added $(P<0.01)$ (Figure 4$)$. Similar results were observed for LIVIN.

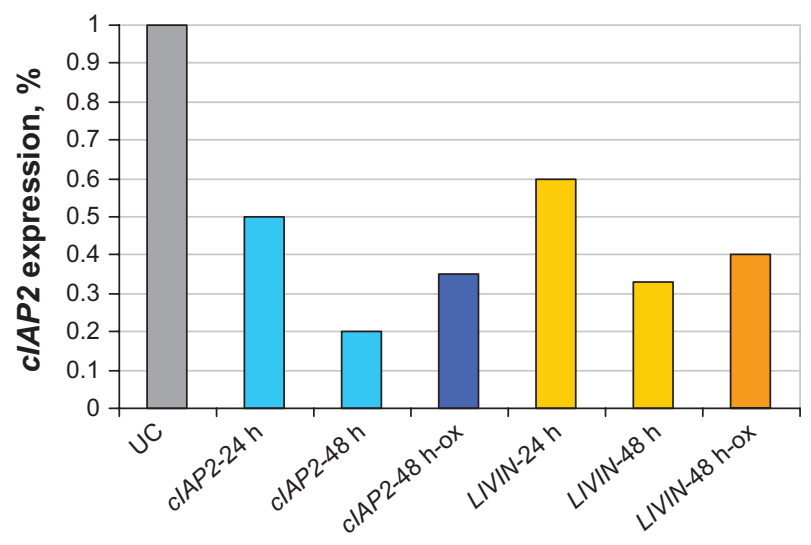

Figure 4 Anti-CIAP2 and anti-LIVIN siRNAs downregulate CIAP2 and LIVIN in HCT116 cells.

Notes: Cells were transfected with anti-cIAP2 or LIVIN siRNA and incubated for 24 and 48 hours ox-free and with ox $(10 \mu \mathrm{M})$. Expression levels were analyzed using real time-PCR.

Abbreviations: h, hours; ox, oxaliplatin; PCR, polymerase chain reaction; siRNA, small interfering RNA; UC, untreated cells.
HCT-116 cells containing $10 \mu \mathrm{M}$ of oxaliplatin were transfected with $15 \mathrm{nM}$ of either anti-cIAP2 or LIVIN siRNAs or both. Scrambled siRNA was added to the cells treated with oxaliplatin in order to control nonspecific inhibition. After 48 hours of incubation, the cells were stained with either Hoechst 33342/propidium iodide (Figure 5A-1a and 2b, B, and C2) or Trypan blue (Figure 5A - 2a and 1b). Apoptotic cells were examined under a fluorescent microscope with ten fields of view per well (10×/20× magnification) (Figure 5). Silencing of each gene resulted in a two-fold increase in sensitivity to oxaliplatin $(P<0.01)$ (Figure 5B) compared to the cells treated with only oxaliplatin (Figure $5 \mathrm{~A}-2 \mathrm{a}$ and $2 \mathrm{~b}$ ). A significant rate of apoptosis $(P<0.005)$ was achieved by
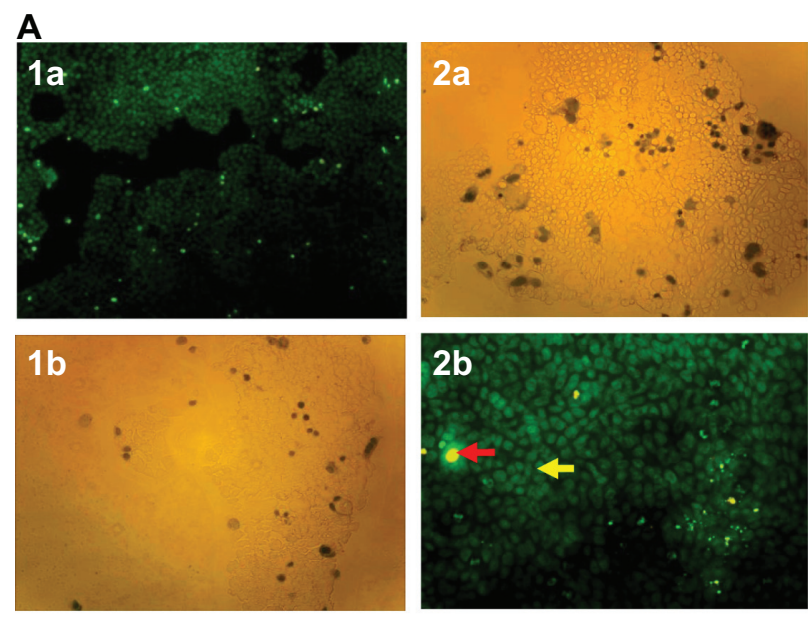

B
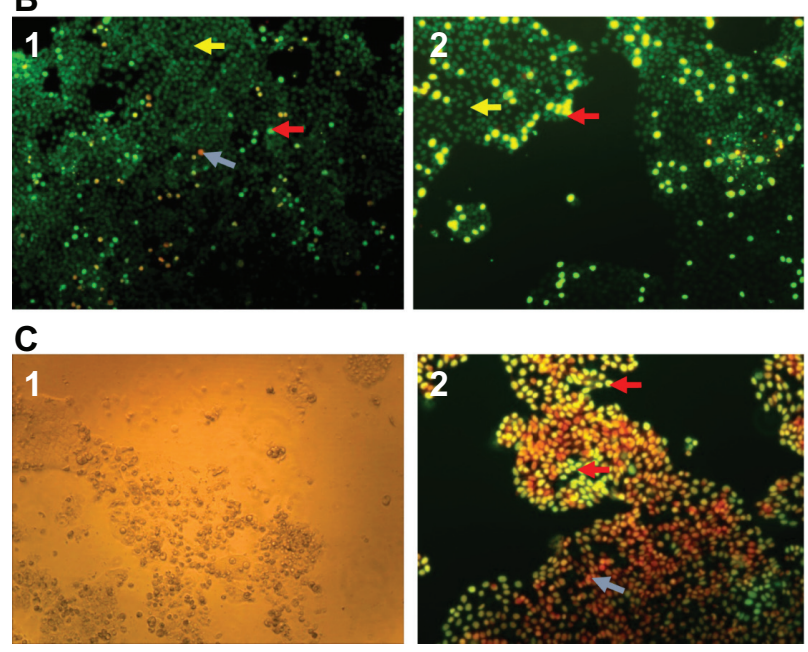

Figure 5 Cells treated with siRNA and oxaliplatin.

Notes: Trypan blue and Hoechst/PI cells were used to detect apoptotic or necrotic cells. (A) la: The untreated cells stained with Hoechst/PI; Ib: the untreated cells stained with Trypane blue. 2a and b: cells treated with $10 \mu \mathrm{M}$ of oxaliplatin. (B) I: Cells treated with cIAP2 + $10 \mu \mathrm{M}$ of oxaliplatin. 2: Cells treated with LIVIN + $10 \mu \mathrm{M}$ of oxaliplatin; (C) I: LIVIN + CIAP2 + $10 \mu \mathrm{M}$ of oxaliplatin treated cells before staining with Hoechst/ PI. 2: Hoechst/PI stained cells treated both with LIVIN + CIAP2 + $10 \mu \mathrm{M}$ of oxaliplatin. All photos made under $10 \times$ magnification. Yellow arrows indicate live cells; blue arrow indicates necrotic red cells; red arrows indicate apoptotic bright green cells. Abbreviations: $\mathrm{PI}$, propidium iodide; siRNA, small interfering RNA. 
means of combination of CIAP2 (Birc3) and LIVIN (Birc7) (Figures 5C and 6).

\section{Discussion}

The separation of gene expression into early and late phases may at first appear to be approximate; however, no genes dependent on NF- $\mathrm{KB}$ signaling were expressed in the first 24 hours, and none (except TRAPl, c-Myc, Bcl-xl and Gstm2) were found to be dose-dependent during this period (Figure 2C). The increase in oxaliplatin dosage, from 5 to $10 \mu \mathrm{M}$, resulted in synergetic elevation of TRAPl and $c-M y c$ at the early stage. This is not surprising as long as TRAPl is considered a direct $c-M y c$ target. The two-fold elevation of Gstm 2 and $B c l-x l$ may be explained by their lack of sensitivity to lower doses of oxaliplatin. $B c l-x l$ maintains its sensitivity only to higher doses of oxaliplatin. Figure $2 \mathrm{C}$ and $\mathrm{D}$ clearly show that TRAP1 is an early-expressed gene, while CIAP2, $F L I P$, and XIAP are the most dose-dependent late genes. Our observations concerning the oxaliplatin sensitivity of $B c l 2$ family members were interesting; a possible explanation for the inactivity of $B c l 2$, compared to the upregulation of $B c l-x l$ expression, is its lack of sensitivity to low concentrations of oxaliplatin.

Due to oxaliplatin's ability to induce DNA damage, there has been much discussion of the role of DNA repair genes in oxaliplatin resistance, such as $E R C C 1$, which has been shown to be inversely correlated with the response to oxaliplatin..$^{12,13}$ However, these findings appear to be controversial, since at least two studies ${ }^{4,14}$ found no association between DNA repair proteins and oxaliplatin resistance. In our experiments, we found $E R C C 1$ to be downregulated in 48 hours at $5 \mu \mathrm{M}$ of

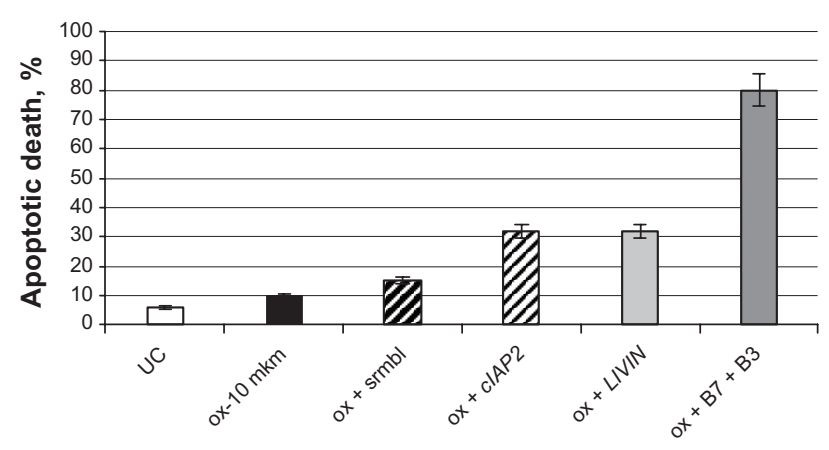

Figure 6 The elevated response to ox of HCT-I 16 cells that were treated with anti-LIVIN and anti-CIAP-2 siRNAs.

Notes: Cells were incubated for 48 hours. Cells were treated with $10 \mu \mathrm{M}$ of ox only (black column) and ox plus either CIAP2, LIVIN, scrambled, or CIAP2 + LIVIN siRNAs. UC were used as negative control. Scrambled siRNA in addition with ox $(10 \mu \mathrm{M})$ resulted in $15 \%$ of cell death. Cocktail silencing of LIVIN (B7) and CIAP-2 (B3) increased sensitivity of cells to ox five-fold compared to Scrambled siRNA in addition with ox $(10 \mu \mathrm{M})$ which caused $15 \%$ cell death.

Abbreviations: ox, oxaliplatin; srmbl, scrambled; siRNA, small interfering RNA; UC, untreated cells. oxaliplatin exposure. Almost full knockdown of Survivin expression was observed at higher doses of oxaliplatin (Figure 2D). The ability of oxaliplatin to downregulate Survivin has been repeatedly described. ${ }^{15-17}$

Our data suggest that CIAP2 and LIVIN play an essential role in the oxaliplatin-induced resistance of HCT116 cells. Unlike $C I A P 2$, LIVIN was less frequently found to be overexpressed in colon cancers. In one very recent study, ${ }^{18}$ anti-LIVIN siRNA apoptosis was observed in vivo and in vitro (in HCT-116 cells), and the authors describe a remarkable decrease in cell viability under different doses of siRNA. One year previously, another group of researchers constructed a vector containing short hairpin RNA-LIVIN that caused apoptosis of HCT-8/V colon cancer cells. ${ }^{19}$ Simultaneous siRNA knockdown of Survivin and LIVIN was performed on Lovo colon cancer cells $;{ }^{20}$ however, no synergic effect was observed. By means of accurate measurement of oxaliplatin-induced expression, we have revealed that $C I A P 2$ and LIVIN were the only genes not downregulated over 48 hours of 5 and $10 \mu \mathrm{M}$ of oxaliplatin exposure. Individual knockdown by means of either anti-LIVIN or CIAP2 resulted in $32 \%$ cell death for both siRNAs. In general, this level of apoptosis is common in cases where only one siRNA is used in order to overcome platinum-containing drug resistance, and has been previously described by several authors. ${ }^{21,22}$ A very representative result was obtained by the previously mentioned group ${ }^{18}$ who performed LIVIN silencing on HCT-116 cells. They observed an approximately $30 \%$ decrease in cell viability with an siRNA concentration of $25 \mathrm{nM}$, which is similar to the concentration used in this study.

Both LIVIN and CIAP2 inhibit caspases 3 and 9, and our study indicated that both are time- and dose-dependent. This finding led us to suppose a synergistic role of these genes in oxaliplatin resistance. As the result of double siRNA silencing, we observed massive apoptosis that made cancer cells so weak and sensitive to ethylenediaminetetraacetic acid (EDTA)-trypsin treatment that cell imaging by means of a microscope was the only way to determine cell viability.

\section{Conclusion}

The siRNA cocktail appears to be an effective tool with which to inhibit several targets that are essential for cancer cell proliferation or survival activity. Over the last 3 years, a number of authors have published results showing a successful cooperative effect in other types of cancers by employing two or three different siRNAs, including CIAP1, CIAP2, and $X I A P$ for prostate cancer ${ }^{23}$ or XIAP, LIVIN, and Survivin 
for bladder cancer. ${ }^{24}$ Together, these findings provide new insight into approaches for the prevention of drug resistance in cancer cells.

\section{Acknowledgments}

We thank Professor Albert Shtill (N.N. Blokhin Cancer Research Center, Moscow) for providing the cell line for this study, and Dr Natalya Pospekhova and Dr Vitaliy Shubin (Centre for Medical Genetics, Moscow) for their support and advice.

\section{Disclosure}

The authors report no conflicts of interest in this work.

\section{References}

1. Samimi G, Manorek G, Castel R, et al. cDNA microarray-based identification of genes and pathways associated with oxaliplatin resistance. Cancer Chemother Pharmacol. 2005;55(1):1-11.

2. Voland C, Bord A, Péleraux A, et al. Repression of cell cycle-related proteins by oxaliplatin but not cisplatin in human colon cancer cells. Mol Cancer Ther. 2006;5(9):2149-2157.

3. Meynard D, Le Morvan V, Bonnet J, Robert J. Functional analysis of the gene expression profiles of colorectal cancer cell lines in relation to oxaliplatin and cisplatin cytotoxicity. Oncol Rep. 2007;7(5):1213-1221.

4. Arnould S, Hennebelle I, Canal P, Bugat R, Guichard S. Cellular determinants of oxaliplatin sensitivity in colon cancer cell lines. Eur J Cancer. 2003;39(1):112-119.

5. Giacchetti S, Perpoint B, Zidani R, et al. Phase III multicenter randomized trial of oxaliplatin added to chronomodulated fluorouracil-leucovorin as first-line treatment of metastatic colorectal cancer. J Clin Oncol. 2000;18: 136-147.

6. Arango D, Wilson AJ, Shi Q, et al. Molecular mechanisms of action and prediction of response to oxaliplatin in colorectal cancer cells. $\mathrm{Br} J$ Cancer. 2004;91(11):1931-1946.

7. Galligan L, Longley DB, McEwan M, Wilson TR, McLaughlin K, Johnston PG. Chemotherapy and TRAIL-mediated colon cancer cell death: the roles of p53, TRAIL receptors, and c-FLIP. Mol Cancer Ther. 2005;4(12):2026-2036.

8. Seetharam RN, Sood A, Basu-Mallick A, Augenlicht LH, Mariadason JM, Goel S. Oxaliplatin resistance induced by ERCC1 up-regulation is abrogated by siRNA-mediated gene silencing in human colorectal cancer cells. Anticancer Res. 2010;30(7):2531-2538.

9. Coller HA, Grandori C, Tamayo P, et al. Expression analysis with oligonucleotide microarrays reveals that MYC regulates genes involved in growth, cell cycle, signaling, and adhesion. Proc Natl Acad Sci U S A. 2000;97(7):3260-3265.
10. Felts SJ, Owen BA, Nguyen P, Trepel J, Donner DB, Toft DO. The hsp90-related protein TRAP1 is a mitochondrial protein with distinct functional properties. J Biol Chem. 2000;275(5):3305-3312.

11. Masuda Y. The role of mitochondrial chaperone tumor necrosis factorassociated protein 1 (TRAPI) in the regulation of apoptosis. Journal of Health Science. 2011;57(1):1-9.

12. Sohn WJ, Lee JW, Park DG. Change in expression of Survivin caused by using oxaliplatin in HCT116 colon cancer cells. J Korean Soc Coloproctol. 2010;26(4):246-253.

13. Shirota Y, Stoehlmacher J, Brabender J, et al. ERCC1 and thymidylate synthase mRNA levels predict survival for colorectal cancer patients receiving combination oxaliplatin and fluorouracil chemotherapy. J Clin Oncol. 2001;19(23):4298-4304.

14. Harradine KA, Kassner M, Chow D, et al. Functional genomics reveals diverse cellular processes that modulate tumor cell response to oxaliplatin. Mol Cancer Res. 2011;9(2):173-182.

15. Fujie Y, Yamamoto H, Ngan CY, et al. Oxaliplatin, a potent inhibitor of survivin, enhances paclitaxel-induced apoptosis and mitotic catastrophe in colon cancer cells. Jpn J Clin Oncol. 2005;35(8):453-463.

16. Ngan CY, Yamamoto H, Takagi A, et al. Oxaliplatin induces mitotic catastrophe and apoptosis in esophageal cancer cells. Cancer Sci. 2008;99(1):129-139.

17. Khan Z, Khan N, Varma AK, et al. Oxaliplatin-mediated inhibition of survivin increases sensitivity of head and neck squamous cell carcinoma cell lines to paclitaxel. Curr Cancer Drug Targets. 2010;10(7): 660-669.

18. Oh BY, Lee RA, Kim KH. siRNA targeting LIVIN decreases tumor in a xenograft model for colon cancer. World J Gastroenterol. 2011;17(20): $2563-2571$.

19. Wang X, Xu J, Ju S, Ni H, Zhu J, Wang H. LIVIN gene plays a role in drug resistance of colon cancer cells. Clin Biochem. 2010;43(7-8): 655-660.

20. Cai M, Wang GB, Tao KX, Cai CX. [Inhibitory effect of multiple siRNA recombinant expression vector targeting simultaneously LIVIN and Survivin genes on human colon cancer cells proliferation.] Yao Xue Хие Bao. 2009;44(7):741-746. Chinese.

21. Zhang S, Ding F, Luo A, et al. XIAP is highly expressed in esophageal cancer and its downregulation by RNAi sensitizes esophageal carcinoma cell lines to chemotherapeutics. Cancer Biol Ther. 2007;6(6): 973-980.

22. Yanagie H, Hisa T, Ogata A, et al. Improvement of sensitivity to platinum compound with siRNA knockdown of upregulated genes in platinum complex-resistant ovarian cancer cells in vitro. Biomed Pharmacother. 2009;63(8):553-560.

23. Gill C, Dowling C, O'Neill AJ, Watson RW. Effects of cIAP-1, cIAP-2 and XIAP triple knockdown on prostate cancer cell susceptibility to apoptosis, cell survival and proliferation. Mol Cancer. 2009;8:39.

24. Yang D, Song X, Zhang J, et al. Therapeutic potential of siRNA-mediated combined knockdown of the IAP genes (LIVIN, XIAP, and Survivin) on human bladder cancer T24 cells. Acta Biochim Biophys Sin (Shanghai). 2010;42(2):137-144.
OncoTargets and Therapy

\section{Publish your work in this journal}

OncoTargets and Therapy is an international, peer-reviewed, open access journal focusing on the pathological basis of all cancers, potential targets for therapy and treatment protocols employed to improve the management of cancer patients. The journal also focuses on the impact of management programs and new therapeutic agents and protocols on

Submit your manuscript here: http://www.dovepress.com/oncotargets-and-therapy-journal

\section{Dovepress}

patient perspectives such as quality of life, adherence and satisfaction. The manuscript management system is completely online and includes a very quick and fair peer-review system, which is all easy to use. Visit http://www.dovepress.com/testimonials.php to read real quotes from published authors. 